\title{
Computing Hypercrossed Complex Pairings in Digital Images
}

\author{
Simge Öztunç, Ali Mutlu, and Necdet Bildik \\ Mathematics Department, Faculty of Science and Arts, Celal Bayar University, 45047 Manisa, Turkey \\ Correspondence should be addressed to Simge Öztunç; simgeoztunc@gmail.com \\ Received 3 October 2013; Accepted 9 November 2013 \\ Academic Editor: Abdon Atangana
}

Copyright (C) 2013 Simge Öztunç et al. This is an open access article distributed under the Creative Commons Attribution License, which permits unrestricted use, distribution, and reproduction in any medium, provided the original work is properly cited.

We consider an additive group structure in digital images and introduce the commutator in digital images. Then we calculate the hypercrossed complex pairings which generates a normal subgroup in dimension 2 and in dimension 3 by using 8-adjacency and 26-adjacency.

\section{Introduction}

In this paper we denote the set of integers by $\mathbb{Z}$. Then $\mathbb{Z}^{n}$ represents the set of lattice points in Euclidean $n$-dimensional spaces. A finite subset of $\mathbb{Z}^{n}$ with an adjacency relation is called a digital image.

Definition 1 (see $[1,2])$. Consider the following.

(1) Two points $p$ and $q$ in $\mathbb{Z}$ are 2-adjacent if $|p-q|=1$.

(2) Two points $p$ and $q$ in $\mathbb{Z}^{2}$ are 8 -adjacent if they are distinct and differ by at most 1 in each coordinate.

(3) Two points $p$ and $q$ in $\mathbb{Z}^{2}$ are 4 -adjacent if they are 8 -adjacent and differ by exactly one coordinate.

(4) Two points $p$ and $q$ in $\mathbb{Z}^{2}$ are 26 -adjacent if they are distinct and differ by at most 1 in each coordinate.

(5) Two points $p$ and $q$ in $\mathbb{Z}^{2}$ are 18-adjacent if they are 26 -adjacent and differ in at most two coordinates.

(6) Two points $p$ and $q$ in $\mathbb{Z}^{2}$ are 6-adjacent if they are 18 -adjacent and differ by exactly one coordinate.

Definition 2. Let $\mathbf{G}$ be a subset of a digital image. A simplicial group $\mathbf{G}$ in digital images consists of a sequence of groups $\mathbf{G}$ and collections of group homomorphisms $d_{i}: G_{n} \rightarrow G_{n-1}$ and $s_{i}: G_{n} \rightarrow G_{n+1}, 0 \leq i \leq n$, that satisfies the following axioms:

$$
\begin{gathered}
d_{i} d_{j}=d_{j-1} d_{i}, \quad i<j, \\
d_{i} s_{j}=s_{j-1} d_{i}, \quad i<j, \\
d_{j} s_{j}=d_{j+1} s_{j}=i d, \quad i=j \text { or } i=j+1, \\
d_{i} s_{j}=s_{j} d_{i-1}, \quad i>j+1, \\
s_{i} s_{j}=s_{j+1} s_{i}, \quad i \leq j .
\end{gathered}
$$

Definition 3. Given a simplicial group $\mathbf{G}$ with $\kappa$-adjacency, the Moore complex $(\mathbf{N G}, \partial$ ) of $\mathbf{G}$ is the chain complex defined by

$$
N G_{n}=\bigcap_{i=0}^{n-1} \operatorname{Ker} d_{i},
$$

with $\partial: N G_{n} \rightarrow N G_{n-1}$ induced from $d_{n}$ by restriction.

The $n$th homology group of the Moore complex of $\mathbf{G}$ is

$$
H_{n}(\mathbf{N G}, \partial)=\frac{\bigcap_{i=0}^{n} \operatorname{Ker} d_{i}}{d_{n+1}\left(\bigcap_{i=0}^{n} \operatorname{Ker} d_{i}\right)} .
$$

\section{Hypercrossed Complex Pairings in Digital Images}

First of all we adapt ideas from Carrasco and Cegarra [3-5] to get the construction in digital images. We define a set $P(n)$ 
consisting of pairs of elements $(\alpha, \beta)$ from $S(n)$ with $\alpha \cap \beta=$ $\varnothing$ and $\beta<\alpha$, with respect to lexicographic ordering in $S(n)$ where $\alpha=\left(i_{l}, \ldots, i_{1}\right)$ and $\beta=\left(j_{m}, \ldots, j_{1}\right) \in S(n)$.

Consider the following diagram:

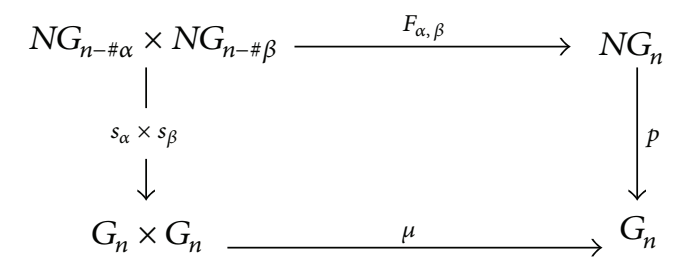

$\left\{F_{\alpha, \beta}: N G_{n-\# \alpha} \times N G_{n-\# \beta} \longrightarrow N G_{n}:(\alpha, \beta) \in P(n), n \geq 0\right\}$

where

$$
\begin{gathered}
s_{\alpha}=s_{i_{l}} \cdots s_{i_{1}}: N G_{n-\# \alpha} \longrightarrow G_{n}, \\
s_{\beta}=s_{j_{m}} \cdots s_{j_{1}}: N G_{n-\# \beta} \longrightarrow G_{n},
\end{gathered}
$$

and define $p: G_{n} \rightarrow N G_{n}$ and $p(x)=p_{n-1} \cdots p_{0}(x)$ as $p_{j}(z)=z-s_{i} d_{i} z$ and $j=0, \ldots, n-1$. Since a digital image has the additive group structure, define the commutator as

$$
[x, y]=x y-y x \text {. }
$$

Thus

$$
\begin{gathered}
\mu: G_{n} \times G_{n} \longrightarrow G_{n}, \\
F_{\alpha, \beta}\left(x_{\alpha}, y_{\beta}\right)=p \mu\left(s_{\alpha} \times s_{\beta}\right)\left(x_{\alpha}, y_{\beta}\right) \\
=p\left[s_{\alpha} x_{\alpha}, s_{\beta} y_{\beta}\right] .
\end{gathered}
$$

The normal subgroup $N G_{n}$ of $G_{n}$ is generated by the elements of the form

$$
F_{\alpha, \beta}\left(x_{\alpha}, y_{\beta}\right)
$$

where $x_{\alpha} \in N G_{n-\# \alpha}$ and $y_{\beta} \in N G_{n-\# \beta}$.

Theorem 4. 2-dimensional normal subgroup $\mathrm{N}_{2}$ with 8-adjacency is generated by the elements of the form

$$
\left[s_{0} x_{1}-s_{1} x_{1}, s_{1} y_{1}\right]
$$

Proof. Let $\alpha=(1)$ and $\beta=(0)$ for $n=2$. For $x_{1}$ and $y_{1} \in$ $N G_{1}=\operatorname{Ker} d_{0}$

$$
\begin{aligned}
F_{(0),(1)}\left(x_{1}, y_{1}\right) & =p_{1} p_{0}\left[s_{0} x_{1}, s_{1} y_{1}\right] \\
& =p_{1}\left\{\left[s_{0} x_{1}, s_{1} y_{1}\right]-s_{0} d_{0}\left[s_{0} x_{1}, s_{1} y_{1}\right]\right\} \\
& =\left[s_{0} x_{1}-s_{1} x_{1}, s_{1} y_{1}\right] .
\end{aligned}
$$

Thus $F_{(0),(1)}\left(x_{1}, y_{1}\right)=\left[s_{0} x_{1}-s_{1} x_{1}, s_{1} y_{1}\right]$ and this is the element generating $N_{2}$ normal subgroups.

Proposition 5. 3-dimensional normal subgroup $\mathrm{N}_{3}$ with 26adjacency is generated by the elements of the following forms: (i) $\left[s_{1} s_{0} x_{1}-s_{0} s_{1} x_{1}, s_{2} y_{2}\right]$,

(ii) $\left[s_{2} s_{0} x_{1}-s_{2} s_{1} x_{1}, s_{1} y_{2}-s_{2} y_{2}\right]$,

(iii) $\left[s_{0} x_{2}-s_{1} x_{2}+s_{2} x_{2}, s_{1} s_{1} y_{1}\right]$

(iv) $\left[s_{1} x_{2}-s_{2} x_{2}, s_{2} y_{2}\right]$,

(v) $\left[s_{0} x_{2}, s_{2} y_{2}\right]$,

(vi) $\left[s_{0} x_{2}-s_{1} x_{2}, s_{1} y_{2}\right]+\left[s_{2} x_{2}, s_{2} y_{2}\right]$.

Proof. For $n=3$ the possible pairings are the following:
(i) $F_{(1,0)(2)}$,
(ii) $F_{(2,0)(1)}$,
(iii) $F_{(0)(2,1)}$,
(iv) $F_{(1)(2)}$,
(v) $F_{(0)(2)}$,
(vi) $F_{(0)(1)}$.

For all $x_{1} \in N G_{1}$ and $y_{2} \in N G_{2}$ the corresponding generators of $N_{3}$ are the following with $F_{\alpha, \beta}: N G_{1} \times N G_{2} \rightarrow N G_{3}$ and for $n=3, p(x)=p_{2} p_{1} p_{0}(x)$ :

(i)

$$
\begin{aligned}
F_{(1,0)(2)}\left(x_{1}, y_{2}\right) & =p\left[s_{1} s_{0} x_{1}, s_{2} y_{2}\right] \\
& =p_{2} p_{1} p_{0}\left[s_{1} s_{0} x_{1}, s_{2} y_{2}\right] \\
& =\left[s_{1} s_{0} x_{1}-s_{2} s_{1} x_{1}, s_{2} y_{2}\right],
\end{aligned}
$$

(ii)

$$
\begin{aligned}
F_{(2,0)(1)}\left(x_{1}, y_{2}\right) & =p\left[s_{2} s_{0} x_{1}, s_{1} y_{2}\right] \\
& =p_{2} p_{1} p_{0}\left[s_{2} s_{0} x_{1}, s_{1} y_{2}\right] \\
& =\left[s_{2} s_{0} x_{1}-s_{2} s_{1} x_{1}, s_{1} y_{2}-s_{2} y_{2}\right] .
\end{aligned}
$$

For all $x_{2} \in N G_{2}$ and $y_{1} \in N G_{1}$ and considering the map $F_{\alpha, \beta}: N G_{2} \times N G_{1} \rightarrow N G_{3}$, the corresponding generator of $N_{3}$ is

(iii)

$$
\begin{aligned}
F_{(0)(2,1)}\left(x_{2}, y_{1}\right) & =p\left[s_{0} x_{2}, s_{2} s_{1} y_{1}\right] \\
& =p_{2} p_{1} p_{0}\left[s_{0} x_{2}, s_{2} s_{1} y_{1}\right] \\
& =\left[s_{0} x_{2}-s_{1} x_{2}+s_{2} x_{2}, s_{2} s_{1} y_{1}\right] .
\end{aligned}
$$

For all $x_{2}, y_{2} \in N G_{2}$ and for $F_{\alpha, \beta}: N G_{2} \times N G_{2} \rightarrow N G_{3}$ the corresponding generators of $N_{3}$ are

(iv)

$$
\begin{aligned}
F_{(1)(2)}\left(x_{2}, y_{2}\right) & =p\left[s_{1} x_{2}, s_{2} y_{2}\right] \\
& =p_{2} p_{1} p_{0}\left[s_{1} x_{2}, s_{2} y_{2}\right] \\
& =\left[s_{1} x_{2}-s_{2} x_{2}, s_{2} y_{2}\right],
\end{aligned}
$$

(v)

$$
\begin{aligned}
F_{(0)(2)}\left(x_{2}, y_{2}\right) & =p\left[s_{0} x_{2}, s_{2} y_{2}\right] \\
& =p_{2} p_{1} p_{0}\left[s_{0} x_{2}, s_{2} y_{2}\right] \\
& =\left[s_{0} x_{2}, s_{2} y_{2}\right],
\end{aligned}
$$


$(\mathrm{vl})$

$$
\begin{aligned}
F_{(0)(1)}\left(x_{2}, y_{2}\right) & =p\left[s_{0} x_{2}, s_{1} y_{2}\right] \\
& =p_{2} p_{1} p_{0}\left[s_{0} x_{2}, s_{1} y_{2}\right] \\
& =\left[s_{0} x_{2}-s_{1} x_{2}, s_{1} y_{2}\right]+\left[s_{2} x_{2}, s_{2} y_{2}\right] .
\end{aligned}
$$

Theorem 6. Let $\mathrm{NG}_{2}$ be a 2-dimensional Moore complex of a simplicial group $\mathbf{G}$. Then $\partial_{2}\left(N G_{2}\right)=\left[\operatorname{Ker} d_{0}, \operatorname{Ker} d_{1}\right]$ where $\partial_{2}$ is induced from $d_{2}$ by restriction.

Proof. For $n=2$, assume that $\alpha=(0), \beta=(1)$, and $x_{1}, y_{1} \in$ $N G_{1}=\operatorname{Ker} d_{0}$. Now calculate $d_{n} F_{\alpha, \beta}$.

Since $F_{(0),(1)}\left(x_{1}, y_{1}\right)=\left[s_{0} x_{1}-s_{1} x_{1}, s_{1} y_{1}\right]$, from Proposition 5

$$
\begin{aligned}
d_{2} F_{(0),(1)}\left(x_{1}, y_{1}\right) & =[d_{2} s_{0} x_{1}-\underbrace{d_{2} s_{1}}_{i d} x_{1}, \underbrace{d_{2} s_{1}}_{i d} y_{1}] \\
& =\left[s_{0} d_{1} x_{1}-x_{1}, y_{1}\right] .
\end{aligned}
$$
not.

At first we investigate whether $s_{0} d_{1} x_{1}-x_{1}$ is in $\operatorname{Ker} d_{0}$ or

$$
\underbrace{d_{0} s_{0}}_{i d} d_{1} x_{1}-\underbrace{d_{0} x_{1}}_{=0}=d_{1} x_{1}
$$

therefore $s_{0} d_{1} x_{1}-x_{1} \notin \operatorname{Ker} d_{0}$.

Secondly we examine whether $s_{0} d_{1} x_{1}-x_{1}$ is in $\operatorname{Ker} d_{1}$ or not.

Since

$\underbrace{d_{1} s_{0}}_{\text {id }} d_{1} x_{1}-\underbrace{d_{1} x_{1}}_{=0}=d_{1} x_{1}-d_{1} x_{1}=0, \quad s_{0} d_{1} x_{1}-x_{1} \in \operatorname{Ker} d_{1}$.

From the assumption $y_{1} \in \operatorname{Ker} d_{0}$, we get

$$
F_{(0),(1)}\left(x_{1}, y_{1}\right) \in\left[\operatorname{Ker} d_{1}, \operatorname{Ker} d_{0}\right] \text {. }
$$

Theorem 7. Let $N G_{3}$ be a 3-dimensional Moore complex of a simplicial group $\mathbf{G}$ with 26-adjacency. Then

$$
\begin{aligned}
\partial_{3}\left(N G_{3}\right) \subseteq & {\left[\operatorname{Ker} d_{2}, \operatorname{Ker} d_{0} \cap \operatorname{Ker} d_{1}\right] } \\
& +\left[\operatorname{Ker} d_{1}, \operatorname{Ker} d_{0} \cap \operatorname{Ker} d_{2}\right] \\
& +\left[\operatorname{Ker} d_{1} \cap \operatorname{Ker} d_{2}, \operatorname{Ker} d_{0}\right] \\
& +\left[\operatorname{Ker} d_{1}, \operatorname{Ker} d_{0}\right] \\
& +\left[\operatorname{Ker} d_{0} \cap \operatorname{Ker} d_{2}, \operatorname{Ker} d_{0} \cap \operatorname{Ker} d_{1}\right] \\
& +\left[\operatorname{Ker} d_{2}, \operatorname{Ker} d_{0} \cap \operatorname{Ker} d_{1}\right],
\end{aligned}
$$

where $\partial_{3}$ is induced from $d_{3}$ by restriction.

Proof. For $n=3$ investigate $d_{n} F_{\alpha, \beta}$ where $x_{1} \in N G_{1}$ and $y_{2} \in$ $N G_{2}=\operatorname{Ker} d_{0} \cap \operatorname{Ker} d_{1}$.
From Proposition 5 we have $F_{(1,0)(2)}\left(x_{1}, y_{2}\right)=\left[s_{1} s_{0} x_{1}-\right.$ $\left.s_{2} s_{0} x_{1}, s_{2} y_{2}\right]$. Then applying $d_{3}$ to $F_{(1,0)(2)}\left(x_{1}, y_{2}\right)$, we get the following:

$$
\begin{aligned}
d_{3} F_{(1,0)(2)}\left(x_{1}, y_{2}\right) & =[d_{3} s_{1} s_{0} x_{1}-\underbrace{d_{3} s_{2} s_{0}}_{i d} x_{1}, \underbrace{d_{3} s_{2}}_{i d} y_{2}] \\
& =\left[s_{1} d_{2} s_{0} x_{1}-s_{0} x_{1}, y_{2}\right] \\
& =\left[s_{1} s_{0} d_{1} x_{1}-s_{0} x_{1}, y_{2}\right] .
\end{aligned}
$$

Firstly, examine whether $s_{1} s_{0} d_{1} x_{1}-s_{0} x_{1}$ is in $\operatorname{Ker} d_{0}$ or not:

$$
\begin{aligned}
d_{0}\left(s_{1} s_{0} d_{1} x_{1}-s_{0} x_{1}\right) & =d_{0} s_{1} s_{0} d_{1} x_{1}-d_{0} s_{0} x_{1} \\
& =s_{0} \underbrace{d_{0} s_{0}}_{\text {id }} d_{1} x_{1}-\underbrace{d_{0} s_{0}}_{\text {id }} x_{1} \\
& =s_{0} d_{1} x_{1}-x_{1} .
\end{aligned}
$$

So $s_{1} s_{0} d_{1} x_{1}-s_{0} x_{1} \notin \operatorname{Ker} d_{0}$.

Secondly we investigate whether $s_{1} s_{0} d_{1} x_{1}-s_{0} x_{1}$ is in Ker $d_{1}$ or not:

$$
\begin{aligned}
d_{1}\left(s_{1} s_{0} d_{1} x_{1}-s_{0} x_{1}\right) & =d_{1} s_{1} s_{0} d_{1} x_{1}-d_{1} s_{0} x_{1} \\
& =\underbrace{d_{1} s_{1} s_{0}}_{\text {id }} d_{1} x_{1}-\underbrace{d_{1} s_{0}}_{\text {id }} x_{1} \\
& =s_{0} d_{1} x_{1}-x_{1} .
\end{aligned}
$$

Therefore $s_{1} s_{0} d_{1} x_{1}-s_{0} x_{1} \notin \operatorname{Ker} d_{1}$.

Finally we check whether $s_{1} s_{0} d_{1} x_{1}-s_{0} x_{1}$ is in Ker $d_{2}$ or not.

Since

$$
\begin{aligned}
d_{2}\left(s_{1} s_{0} d_{1} x_{1}-s_{0} x_{1}\right) & =\underbrace{d_{2} s_{1}}_{i d} s_{0} d_{1} x_{1}-d_{2} s_{0} x_{1} \\
& =s_{0} d_{1} x_{1}-s_{0} d_{1} x_{1}=0,
\end{aligned}
$$

therefore $s_{1} s_{0} d_{1} x_{1}-s_{0} x_{1} \in \operatorname{Ker} d_{2}$.

We get

$$
F_{(0,1),(2)}\left(x_{1}, y_{1}\right) \in\left[\operatorname{Ker} d_{2}, \operatorname{Ker} d_{0} \cap \operatorname{Ker} d_{1}\right],
$$

since $y_{2} \in \operatorname{Ker} d_{0} \cap \operatorname{Ker} d_{1}$.

$$
\begin{aligned}
& \text { If } \\
& F_{(2,0)(1)}\left(x_{1}, y_{2}\right)=\left[s_{2} s_{0} x_{1}-s_{2} s_{1} x_{1}, s_{1} y_{2}-s_{2} y_{2}\right],
\end{aligned}
$$

then

$$
\begin{aligned}
d_{3} & F_{(2,0)(1)}\left(x_{1}, y_{2}\right) \\
& =d_{3}\left(\left[s_{2} s_{0} x_{1}-s_{2} s_{1} x_{1}, s_{1} y_{2}-s_{2} y_{2}\right]\right) \\
& =[\underbrace{d_{3} s_{2} s_{0}}_{i d} x_{1}-\underbrace{d_{3} s_{2} s_{1}}_{i d} x_{1}, d_{3} s_{1} y_{2}-\underbrace{d_{3} s_{2}}_{i d} y_{2}] \\
& =\left[s_{0} x_{1}-s_{1} x_{1}, s_{1} d_{2} y_{2}-y_{2}\right] .
\end{aligned}
$$


At first we check whether $s_{0} x_{1}-s_{1} x_{1}$ is in $\operatorname{Ker} d_{0}, \operatorname{Ker} d_{1}$, and $\operatorname{Ker} d_{2}$ or not.

$$
d_{0}\left(s_{0} x_{1}-s_{1} x_{1}\right)=\underbrace{d_{0} s_{0}}_{\text {id }} x_{1}-d_{0} s_{1} x_{1}=x_{1}-d_{0} s_{1} x_{1} .
$$

Thus $s_{0} x_{1}-s_{1} x_{1} \notin \operatorname{Ker} d_{0}$.

Next, since

$$
\begin{gathered}
d_{1}\left(s_{0} x_{1}-s_{1} x_{1}\right)=\underbrace{d_{1} s_{0}}_{i d} x_{1}-\underbrace{d_{1} s_{1}}_{i d} x_{1}=x_{1}-x_{1}=0, \\
s_{0} x_{1}-s_{1} x_{1} \in \operatorname{Ker} d_{1},
\end{gathered}
$$

and, finally,

$$
d_{2}\left(s_{0} x_{1}-s_{1} x_{1}\right)=d_{2} s_{0} x_{1}-\underbrace{d_{2} s_{1}}_{i d} x_{1}=s_{0} d_{1} x_{1}-x_{1} \notin \operatorname{Ker} d_{2} .
$$

Now examine whether $s_{1} d_{2} y_{2}-y_{2}$ is in $\operatorname{Ker} d_{0}$, Ker $d_{1}$, and Ker $d_{2}$ or not:

$$
\begin{aligned}
d_{0}\left(s_{1} d_{2} y_{2}-y_{2}\right) & =d_{0} s_{1} d_{2} y_{2}-d_{0} y_{2} \\
& =s_{0} d_{0} d_{2} y_{2}-d_{0} y_{2} \\
& =s_{0} d_{1} \underbrace{d_{0} y_{2}}_{=0}-\underbrace{d_{0} y_{2}}_{=0}=0 .
\end{aligned}
$$

Therefore $s_{1} d_{2} y_{2}-y_{2} \in \operatorname{Ker} d_{0}$. We have the following:

$$
\begin{aligned}
& d_{1}\left(s_{1} d_{2} y_{2}-y_{2}\right)=\underbrace{d_{1} s_{1}}_{i d} d_{2} y_{2}-\underbrace{d_{1} y_{2}}_{=0}=d_{2} y_{2} \notin \operatorname{Ker} d_{1} ; \\
& d_{2}\left(s_{1} d_{2} y_{2}-y_{2}\right)=\underbrace{d_{2} s_{1}}_{\text {id }} d_{2} y_{2}-d_{2} y_{2}=d_{2} y_{2}-d_{2} y_{2}=0 \\
& \Longrightarrow s_{1} d_{2} y_{2}-y_{2} \in \operatorname{Ker} d_{2} \text {. }
\end{aligned}
$$

So $F_{(2,0)(1)}\left(x_{1}, y_{2}\right)=\left[\operatorname{Ker} d_{1}, \operatorname{Ker} d_{0} \cap \operatorname{Ker} d_{2}\right]$.

For all $x_{2} \in N G_{2}$ and $y_{1} \in N G_{1}$ if

$$
F_{(0)(2,1)}\left(x_{2}, y_{1}\right)=\left[s_{0} x_{2}-s_{1} x_{2}+s_{2} x_{2}, s_{1} s_{1} y_{1}\right] \text {, }
$$

then

$$
\begin{aligned}
& d_{3} F_{(0)(2,1)}\left(x_{2}, y_{1}\right) \\
& \quad=d_{3}\left(\left[s_{0} x_{2}-s_{1} x_{2}+s_{2} x_{2}, s_{2} s_{1} y_{1}\right]\right) \\
& \quad=[d_{3} s_{0} x_{2}-d_{3} s_{1} x_{2}+\underbrace{d_{3} s_{2}}_{i d} x_{2}, \underbrace{d_{3} s_{2}}_{i d} s_{1} y_{1}] \\
& \quad=\left[s_{0} d_{2} x_{2}-s_{1} d_{2} x_{2}+x_{2}, s_{1} y_{1}\right] .
\end{aligned}
$$

Firstly investigate whether $s_{0} d_{2} x_{2}-s_{1} d_{2} x_{2}+x_{2}$ is in Ker $d_{0}$, $\operatorname{Ker} d_{1}$, and $\operatorname{Ker} d_{2}$ or not:

$$
\begin{aligned}
& d_{0}\left(s_{0} d_{2} x_{2}-s_{1} d_{2} x_{2}+x_{2}\right) \\
& =\underbrace{d_{0} s_{0}}_{i d} d_{2} x_{2}-d_{0} s_{1} d_{2} x_{2}+\underbrace{d_{0} x_{2}}_{=0} \\
& =d_{2} x_{2}-s_{0} d_{1} \underbrace{d_{0} x_{2}}_{=0}=d_{2} x_{2} .
\end{aligned}
$$

Thereby $s_{0} d_{2} x_{2}-s_{1} d_{2} x_{2}+x_{2} \notin \operatorname{Ker} d_{0}$. We have

$$
\begin{aligned}
& d_{1}\left(s_{0} d_{2} x_{2}-s_{1} d_{2} x_{2}+x_{2}\right) \\
& =\underbrace{d_{1} s_{0}}_{i d} d_{2} x_{2}-\underbrace{d_{1} s_{1}}_{i d} d_{2} x_{2}+\underbrace{d_{1} x_{2}}_{=0} \\
& =d_{2} x_{2}-d_{2} x_{2}=0 .
\end{aligned}
$$

For this reason $s_{0} d_{2} x_{2}-s_{1} d_{2} x_{2}+x_{2} \in \operatorname{Ker} d_{1}$. We also have

$$
\begin{aligned}
& d_{2}\left(s_{0} d_{2} x_{2}-s_{1} d_{2} x_{2}+x_{2}\right) \\
& =d_{2} s_{0} d_{2} x_{2}-\underbrace{d_{2} s_{1}}_{i d} d_{2} x_{2}+d_{2} x_{2} \\
& =s_{0} d_{1} d_{2} x_{2}-d_{2} x_{2}+d_{2} x_{2} \\
& =s_{0} d_{1} \underbrace{d_{1} x_{2}}_{=0}=0 .
\end{aligned}
$$

Hence $s_{0} d_{2} x_{2}-s_{1} d_{2} x_{2}+x_{2} \in \operatorname{Ker} d_{2}$.

Later on we research whether $s_{2} s_{1} y_{1}$ is in $\operatorname{Ker} d_{0}, \operatorname{Ker} d_{1}$, and $\operatorname{Ker} d_{2}$ or not.

Since $d_{0}\left(s_{1} y_{1}\right)=s_{0} \underbrace{d_{0} y_{1}}_{=0}=0, s_{1} y_{1} \in \operatorname{Ker} d_{0}$.

Since $d_{1}\left(s_{1} y_{1}\right)=\underbrace{d_{1} s_{1}}_{\text {id }} y_{1}=y_{1}, s_{1} y_{1} \notin \operatorname{Ker} d_{1}$.

Since $d_{2}\left(s_{1} y_{1}\right)=\underbrace{d_{2} s_{1}}_{i d} y_{1}=y_{1}, s_{1} y_{1} \notin \operatorname{Ker} d_{2}$.

Thus $d_{3} F_{(0)(2,1)}\left(x_{2}, y_{1}\right) \in\left[\operatorname{Ker} d_{1} \cap \operatorname{Ker} d_{2}, \operatorname{Ker} d_{0}\right]$.

For all $x_{2}, y_{2} \in N G_{2}$ since $F_{(0)(2)}\left(x_{2}, y_{2}\right)=\left[s_{0} x_{2}, s_{2} y_{2}\right]$,

$$
\begin{aligned}
d_{3} F_{(0)(2)}\left(x_{2}, y_{2}\right) & =d_{3}\left(\left[s_{0} x_{2}, s_{2} y_{2}\right]\right) \\
& =[d_{3} s_{0} x_{2}, \underbrace{d_{3} s_{2} y_{2}}_{i d}] \\
& =\left[s_{0} d_{2} x_{2}, y_{2}\right] .
\end{aligned}
$$

By using properties of the commutator we have

$$
\begin{aligned}
& {\left[s_{0} d_{2} x_{2}-s_{1} d_{2} x_{2}+x_{2}, y_{2}\right]} \\
& =\left[s_{0} d_{2} x_{2}+\left(x_{2}-s_{1} d_{2} x_{2}\right), y_{2}\right] \\
& \quad=\left[s_{0} d_{2} x_{2}, y_{2}\right]+\left[x_{2}-s_{1} d_{2} x_{2}, y_{2}\right], \\
& {\left[s_{0} d_{2} x_{2}-s_{1} d_{2} x_{2}+x_{2}, y_{2}\right]+\left[y_{2}, x_{2}-s_{1} d_{2} x_{2}\right]} \\
& =\left[s_{0} d_{2} x_{2}, y_{2}\right] \\
& =d_{3} F_{(0)(2)}\left(x_{2}, y_{2}\right) .
\end{aligned}
$$

Thus

$$
\begin{aligned}
d_{3} F_{(0)(2)}\left(x_{2}, y_{2}\right) & \\
\in & {\left[\operatorname{Ker} d_{1} \cap \operatorname{Ker} d_{2}, \operatorname{Ker} d_{1} \cap \operatorname{Ker} d_{0}\right] } \\
& +\left[\operatorname{Ker} d_{0} \cap \operatorname{Ker} d_{1}, \operatorname{Ker} d_{0} \cap \operatorname{Ker} d_{2}\right] .
\end{aligned}
$$


If $F_{(1)(2)}\left(x_{2}, y_{2}\right)=\left[s_{1} x_{2}-s_{2} x_{2}, s_{2} y_{2}\right]$, then

$$
\begin{aligned}
d_{3} F_{(1)(2)}\left(x_{2}, y_{2}\right) & =d_{3}\left(\left[s_{1} x_{2}-s_{2} x_{2}, s_{2} y_{2}\right]\right) \\
& =[d_{3} s_{1} x_{2}-\underbrace{d_{3} s_{2}}_{\text {id }} x_{2}, \underbrace{d_{3} s_{2}}_{\text {id }} y_{2}] \\
& =\left[s_{1} d_{2} x_{2}-x_{2}, y_{2}\right] .
\end{aligned}
$$

Firstly we check whether $s_{1} d_{2} x_{2}-x_{2}$ is in $\operatorname{Ker} d_{0}$, Ker $d_{1}$, and Ker $d_{2}$ or not:

$$
\begin{aligned}
d_{0}\left(s_{1} d_{2} x_{2}-x_{2}\right) & =d_{0} s_{1} d_{2} x_{2}-\underbrace{d_{0} x_{2}}_{=0} \\
& =s_{0} d_{0} d_{2} x_{2} \\
& =s_{0} d_{1} \underbrace{d_{0} x_{2}}_{=0} \\
& =0 .
\end{aligned}
$$

Therefore $s_{1} d_{2} x_{2}-x_{2} \in \operatorname{Ker} d_{0}$. Since

$$
\begin{aligned}
d_{1}\left(s_{1} d_{2} x_{2}-x_{2}\right) & =\underbrace{d_{1} s_{1}}_{\text {id }} d_{2} x_{2}-\underbrace{d_{1} x_{2}}_{=0} \\
& =d_{2} x_{2},
\end{aligned}
$$

$s_{1} d_{2} x_{2}-x_{2} \notin \operatorname{Ker} d_{1}$. We have

$$
\begin{aligned}
d_{2}\left(s_{1} d_{2} x_{2}-x_{2}\right) & =\underbrace{d_{2} s_{1}}_{i d} d_{2} x_{2}-d_{2} x_{2} \\
& =d_{2} x_{2}-d_{2} x_{2} \\
& =0 .
\end{aligned}
$$

Hence $s_{1} d_{2} x_{2}-x_{2} \in \operatorname{Ker} d_{2}$.

Because of the case $y_{2} \in \operatorname{Ker} d_{0} \cap \operatorname{Ker} d_{1}$,

$$
d_{3} F_{(1)(2)}\left(x_{2}, y_{2}\right) \in\left[\operatorname{Ker} d_{0} \cap \operatorname{Ker} d_{2}, \operatorname{Ker} d_{0} \cap \operatorname{Ker} d_{1}\right] \text {. }
$$

$$
\begin{aligned}
& \text { If } F_{(0)(1)}\left(x_{2}, y_{2}\right)=\left[s_{0} x_{2}-s_{1} x_{2}, s_{1} y_{2}\right]+\left[s_{2} x_{2}, s_{2} y_{2}\right] \text {, then } \\
& \qquad \begin{aligned}
d_{3} F_{(0)(1)}\left(x_{2}, y_{2}\right) \\
\quad=\left[d_{3} s_{0} x_{2}-d_{3} s_{1} x_{2}, s_{1} y_{2}\right]+[\underbrace{d_{3} s_{2}}_{i d} x_{2}, \underbrace{d_{3} s_{2}}_{\text {id }} y_{2}] \\
=\left[s_{0} d_{2} x_{2}-s_{1} d_{2} x_{2}, s_{1} d_{2} y_{2}\right]+\left[x_{2}, y_{2}\right] .
\end{aligned}
\end{aligned}
$$

Consider the following commutator:

$$
\left[s_{0} d_{2} x_{2}-s_{1} d_{2} x_{2}+x_{2}, s_{1} d_{2} y_{2}-y_{2}\right]
$$

and code the terms of this commutator such as

$$
\begin{gathered}
a=s_{0} d_{2} x_{2}, \quad b=s_{1} d_{2} y_{2}, \\
c=s_{1} d_{2} x_{2}, \quad d=x_{2}, \quad e=y_{2},
\end{gathered}
$$

in order to simplify the algebraic operations. Thus, by using the properties and definition of the commutator we obtain the following:

$$
\begin{gathered}
{[a-c+d, b-e]=[a-c, b]+[d, e],} \\
(a-c+d)(b-e)-(b-e)(a-c+d) \\
=a b-c b+d b-a e+c e-d e \\
-\{b a-b c+b d-e a+e c-e d\} .
\end{gathered}
$$

Consider the following cases:

$$
\begin{aligned}
a b-c b-b a+b c & =(a-c) b-b(a-c) \\
& =[a-c, b], \\
c e-d e-e c+e d & =(c-d) e-e(c-d) \\
& =[c-d, e] .
\end{aligned}
$$

And from the remaining terms we get

$$
\begin{aligned}
d b-b d-[d, e] & =d b-b d-d e+e d \\
& =d(b-e)-(b-e) d \\
& =[d, b-e] .
\end{aligned}
$$

Consequently for $n=3$ we have

$$
\begin{aligned}
\partial_{3}\left(N G_{3}\right) \subseteq & {\left[\operatorname{Ker} d_{2}, \operatorname{Ker} d_{0} \cap \operatorname{Ker} d_{1}\right] } \\
& +\left[\operatorname{Ker} d_{1}, \operatorname{Ker} d_{0} \cap \operatorname{Ker} d_{2}\right] \\
& +\left[\operatorname{Ker} d_{1} \cap \operatorname{Ker} d_{2}, \operatorname{Ker} d_{0}\right] \\
& +\left[\operatorname{Ker} d_{1}, \operatorname{Ker} d_{0}\right] \\
& +\left[\operatorname{Ker} d_{0} \cap \operatorname{Ker} d_{2}, \operatorname{Ker} d_{0} \cap \operatorname{Ker} d_{1}\right] \\
& +\left[\operatorname{Ker} d_{2}, \operatorname{Ker} d_{0} \cap \operatorname{Ker} d_{1}\right] .
\end{aligned}
$$

Corollary 8. Let $\mathrm{NG}_{3}$ be a 3-dimensional Moore complex of a simplicial group $\mathbf{G}$ with 26-adjacency. Then

$$
\begin{aligned}
\partial_{3}\left(N G_{3}\right) \subseteq & {\left[\operatorname{Ker} d_{2}, \operatorname{Ker} d_{0} \cap \operatorname{Ker} d_{1}\right] } \\
& +\left[\operatorname{Ker} d_{1}, \operatorname{Ker} d_{0} \cap \operatorname{Ker} d_{2}\right] \\
& +\left[\operatorname{Ker} d_{1} \cap \operatorname{Ker} d_{2}, \operatorname{Ker} d_{0}\right] \\
& +\left[\operatorname{Ker} d_{1}, \operatorname{Ker} d_{0}\right] \\
& +\left[\operatorname{Ker} d_{0} \cap \operatorname{Ker} d_{2}, \operatorname{Ker} d_{0} \cap \operatorname{Ker} d_{1}\right] \\
& +\left[\operatorname{Ker} d_{2}, \operatorname{Ker} d_{0} \cap \operatorname{Ker} d_{1}\right] .
\end{aligned}
$$


Proof. Otherwise inclusion for the previous theorem is obtained from $[4,5]$. Therefore

$$
\begin{aligned}
\partial_{3}\left(N G_{3}\right)= & {\left[\operatorname{Ker} d_{2}, \operatorname{Ker} d_{0} \cap \operatorname{Ker} d_{1}\right] } \\
& +\left[\operatorname{Ker} d_{1}, \operatorname{Ker} d_{0} \cap \operatorname{Ker} d_{2}\right] \\
& +\left[\operatorname{Ker} d_{1} \cap \operatorname{Ker} d_{2}, \operatorname{Ker} d_{0}\right] \\
& +\left[\operatorname{Ker} d_{1}, \operatorname{Ker} d_{0}\right] \\
& +\left[\operatorname{Ker} d_{0} \cap \operatorname{Ker} d_{2}, \operatorname{Ker} d_{0} \cap \operatorname{Ker} d_{1}\right] \\
& +\left[\operatorname{Ker} d_{2}, \operatorname{Ker} d_{0} \cap \operatorname{Ker} d_{1}\right] .
\end{aligned}
$$

\section{Conclusion}

In this paper for dimension 2 and dimension 3, we obtained the Moore complex of simplicial groups generated by hypercrossed complex pairings in digital images.

\section{References}

[1] L. Boxer, "Digitally continuous functions," Pattern Recognition Letters, vol. 15, no. 8, pp. 833-839, 1994.

[2] I. Karaca, L. Boxer, and A. Öztel, "Topological Invariants in digital images," Journal of Mathematical Science, vol. 11, no. 2, pp. 109-140, 2011.

[3] P. Carrasco and A. M. Cegarra, "Group-theoretic algebraic models for homotopy types," Journal of Pure and Applied Algebra, vol. 75, no. 3, pp. 195-235, 1991.

[4] A. Mutlu, Peiffer pairings in the moore complex of a simplicial group [Ph.D. thesis], University of Walles, Bangor, UK, 1997.

[5] A. Mutlu and T. Porter, "Application of Peiffer pairings in the Moore complex of a simplicial Group," Theory and Applications of Categories, vol. 4, no. 7, pp. 148-173, 1998. 


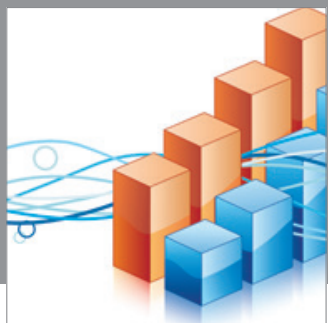

Advances in

Operations Research

mansans

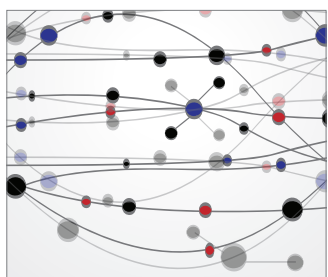

The Scientific World Journal
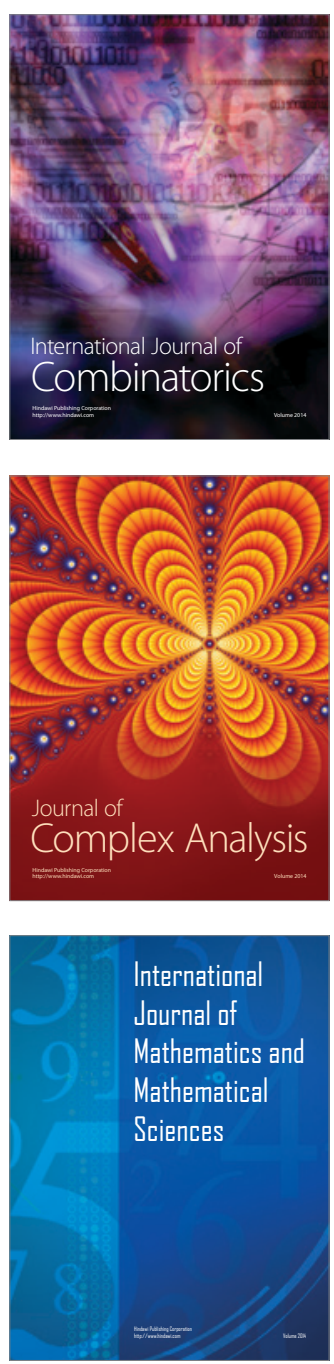
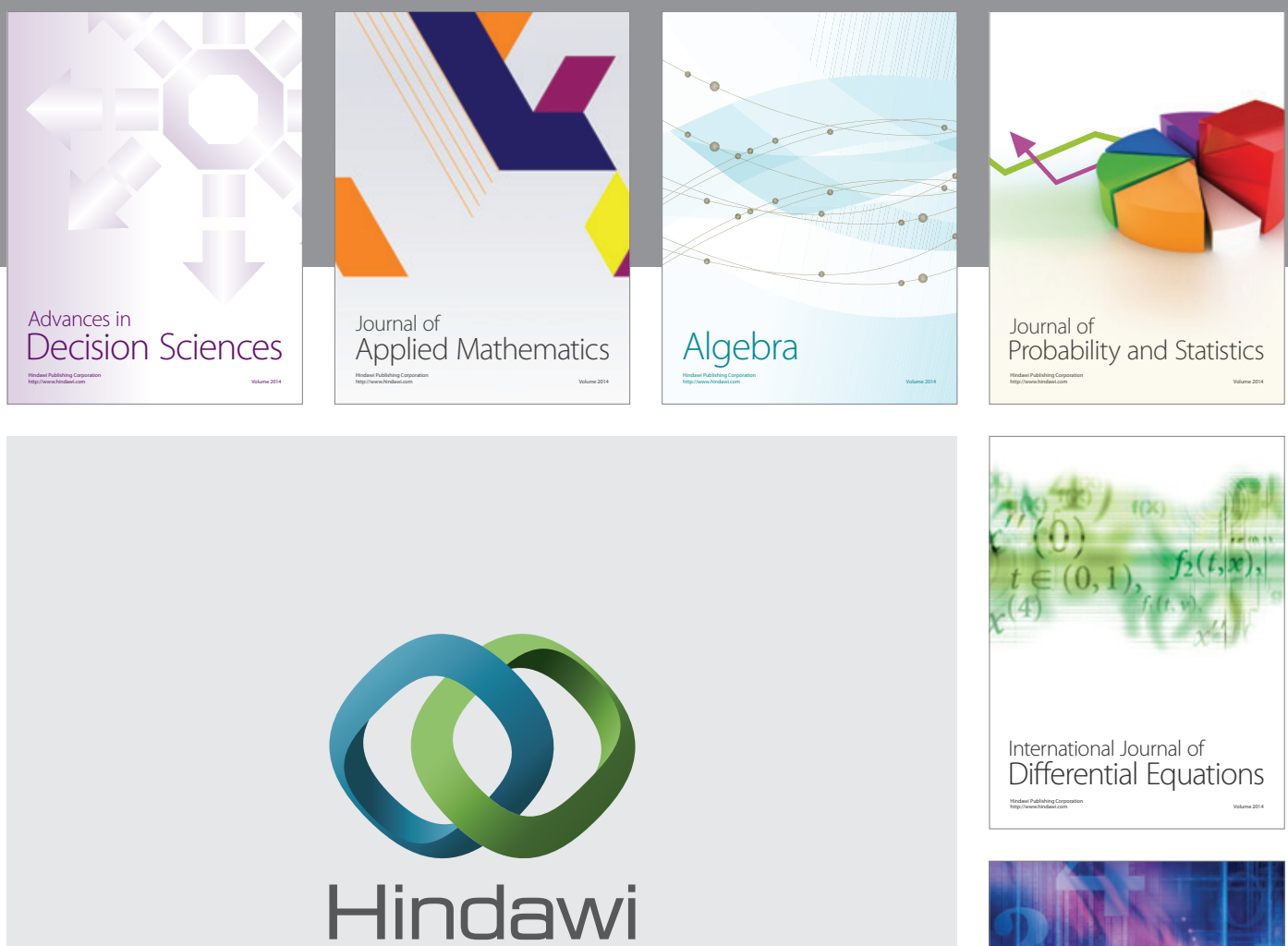

Submit your manuscripts at http://www.hindawi.com
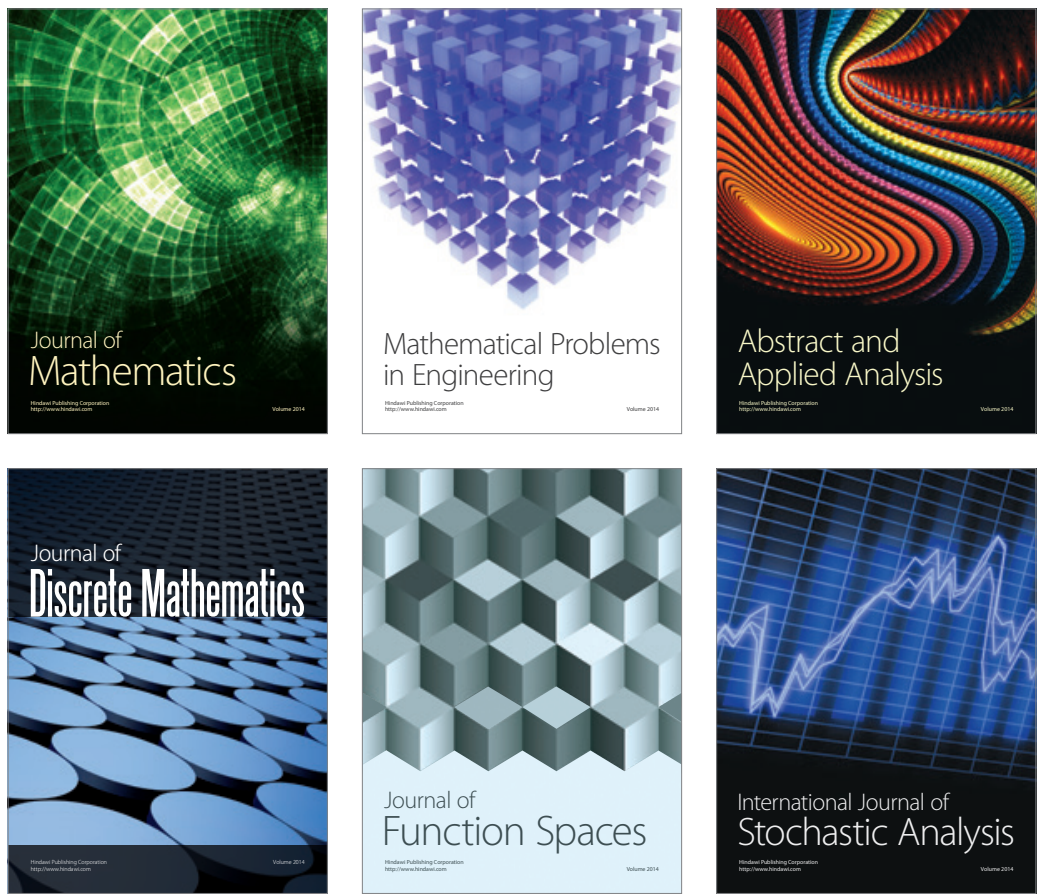

Journal of

Function Spaces

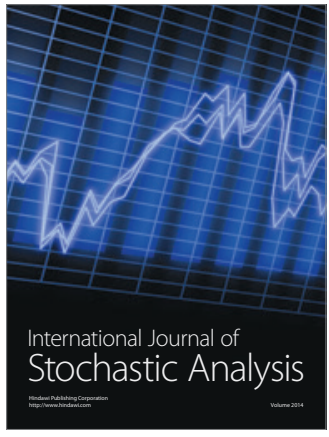

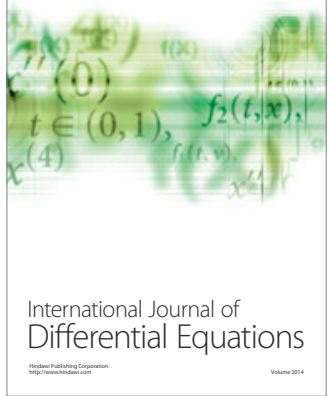
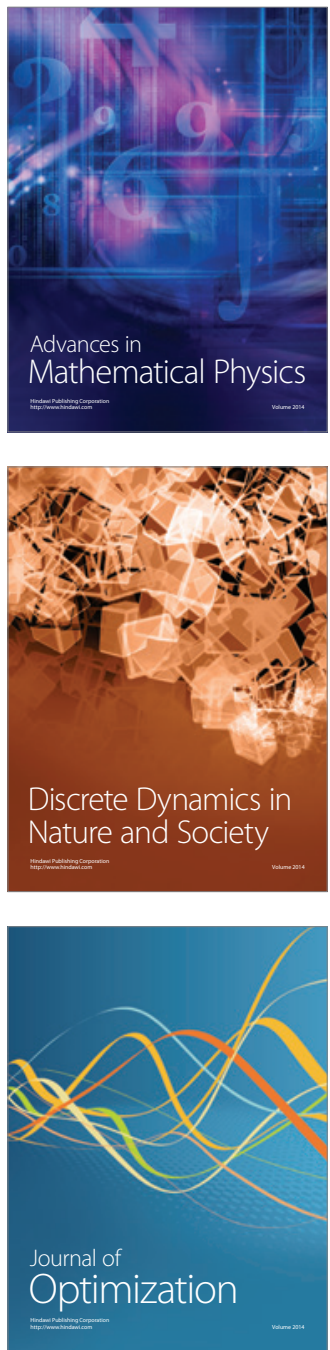